\title{
Are Antibiotics Prescribed to Inpatients According to Recommended Standard Guidelines in Juba Teaching Hospital, South Sudan? A Retrospective Cross-sectional Study
}

\author{
Michael Ekubu Otim ( $\nabla$ motim@sharjah.ac.ae) \\ University of Sharjah College of Health Sciences https://orcid.org/0000-0001-9005-5651 \\ Desmond Kabuya Demaya \\ Nexus International University, Uganda \\ Amina M Al-Marzouqi \\ University of Sharjah College of Health Sciences \\ Jean P. Mukasa
}

Fatima College of Health Sciences

\section{Research}

Keywords: Antibiotics, Low-income countries, Clinical guidelines, Policy implications, Antimicrobial resistance (AMR), Antibiotic use

Posted Date: April 8th, 2021

DOl: https://doi.org/10.21203/rs.3.rs-401614/v1

License: (9) This work is licensed under a Creative Commons Attribution 4.0 International License.

Read Full License 


\section{Abstract}

\section{Background:}

The emergence and spread of antibiotic resistance occurred at an alarming rate globally, and has limited the use of antibiotics for preventing and treating infectious diseases. Failure to follow guidelines and protocols for antibiotic use for rational therapeutics and infection control has led to overuse and misuse of antibiotics. However, there is paucity of data on the current pattern of antibiotics prescribing for hospitalised patients in South Sudan, which is among the youngest countries in the world. Therefore, this study aimed to explore the prescription and use of antibiotics at Juba Teaching Hospital (JTH) in South Sudan.

\section{Methods:}

A retrospective cross-sectional study was conducted among JTH inpatients between January and December 2016. A sample of 316 files from the medical and surgical wards were extracted using probability sampling proportional to ward size. Extracted files were reviewed to determine the pattern of antibiotics administration. Data on indications for antibiotic use were also collected. All data were entered twice and analysed using STATA version 13.0.

\section{Results:}

In all reviewed files, patients received treatment with antibiotics. Antibiotic use was highest in the medical ward (75.4\%). Conditions in which antibiotic use was most prevalent were infectious and parasitic diseases $(23.7 \%)$ and diseases of the digestive system (19.9\%). The top three antibiotics prescribed were ceftriaxone (21.2\%), metronidazole (20.0\%) and amoxicillin (11.5\%). The majority of patients $(40.2 \%)$ were treated with two antibiotics.

\section{Conclusions:}

This study revealed a high level of inappropriate antibiotics use at JJTH. Furthermore, there is nonadherence to national/international guidelines in administration of these antibiotics among doctors. It is necessary to introduce antibiotic stewardship activity, along with continuous national surveillance and enforcement to reduce irrational antibiotics use and the associated risk for antibiotic resistance.

\section{Background}

Globally, there is a burden of antimicrobial resistance (AMR), which is exposing the world to potentially severe superbugs. AMR is mostly attributed to malpractice in prescribing antibiotics to patients without due indication, and accounts for around 700,000 deaths per year [1]. O'Neill [2] estimated that by 2050, the cost of AMR will be approximately 10 million lives lost and US\$100 trillion per year worldwide. Further, Gould et al. and Bartlett et al. estimated that nearly 444 million people would have died because of multidrug resistance (MDR) by 2050 [3] [4]. The use of antimicrobials in the food and livestock industries 
has significantly contributed to AMR [5]. However, recent evaluations of resistance-associated costs have restricted scope, meaning further prospective studies must be conducted to estimate the actual economic burden.

The magnitude of AMR in Africa is not fully understood because of a lack of quality data and weak AMR surveillance systems. However, Essack et al. noted that the high burden of communicable diseases in Africa engenders extensive use of antibiotics (both prescribed and over-the-counter), which may lead to AMR and subsequent costs, health effects, and financial and societal implications [6]. For example, a study in Ghana [7] reported high bacterial resistance to certain drugs including chloramphenicol, tetracycline, ampicillin and co-trimoxazole (>70\%).

To solve the problem of AMR, the World Health Organization [8] advocated for the rational use of drugs based on the 'Rule of Right'. This means that the right drug must be given to the right patient at the right time in the right dose [9]. It has been suggested that this rule is often ignored by clinicians in Africa, leading to an increased risk for AMR. In South Sudan, most healthcare services are publicly funded and provided; therefore, antibiotics use is limited by the prescription of and over-the-counter access to antibiotics.

\section{Theoretical Foundation for this Study}

In theory, microbes develop AMR through a natural selection process wherein some microbes succumb to an antibiotic whereas others do not, meaning these organisms either pass on their resistance genes to their offspring by replication or other related bacteria through 'conjugation' [10]. This process is often exacerbated by the overuse or underuse of antimicrobials in treating human illnesses.

Since its discovery after the world war, II AMR has become a serious public health concern with global economic, social and political implications that cross environmental and ethnic boundaries [10]. AMR occurs when microbes (bacteria, viruses, fungi and parasites) no longer respond to the drugs designed to kill them, and is a public health concern responsible for high mortality and life-threatening infections worldwide [11]. Generally, first- and second-line antibiotic treatment options are limited by resistance and availability of effective antibiotics. Patients with resistant infections have an increased likelihood of dying and survivors have extended hospital stays, delayed recuperation and long-term disability [12]. Achievements in reducing mortality and morbidity through the early use of antibiotics based on empirical guidelines will be jeopardised if appropriate actions are not taken to control AMR [13].

\section{Justification for this study}

In developing countries, it is estimated that $60 \%$ of medicines in public health facilities and $70 \%$ of medicines in private facilities are prescribed and sold inappropriately, resulting in enormous wastage of health resources [14]. Antibiotics are also being used in food production (animals and plants) to prevent, control and treat disease and promote growth [12]. Therefore, the misuse or overuse of these antibiotics increases the chance of the development of MDR bacteria, which contributes to mortality globally. For 
example, approximately 63,000 patients die each year from hospital-acquired bacterial infections in the US, and an estimated 25,000 patients die each year in Europe [12].

MDR bacteria are associated with significant healthcare costs and productivity losses [11] . The loss of effective antibiotics undermines people's ability to fight infectious diseases and manage complications. This is an important concern, particularly among vulnerable patients undergoing chemotherapy for cancer, dialysis for renal failure and surgery (e.g. organ transplantation) where the ability to treat secondary infections is crucial [12]. Bugnon-Reber et al. found that $47 \%$ of antibiotics prescribed in 1 day in surgical and medical wards in a Swiss hospital were inappropriate, and $17(28 \%)$ lacked any indication for antibiotic use [15]. That study also found higher rates of misuse in surgery than in medicine (58\% vs. $34 \%$; odds ratio $[\mathrm{OR}]=2.5,95 \%$ confidence interval $[\mathrm{Cl}]: 1.1-5.9)$, and higher rates for prophylaxis than treatment (72\% vs. $41 \%$; OR=4.1, 95\% Cl: $1.3-15.5$ ). The Centers for Disease Control and Prevention (CDC) estimated that up to $50 \%$ of all prescribed antibiotics are either not needed or inappropriate [16] [17].

Recent studies reported increased antibiotic abuse in developing countries, where irrational antibiotics use has become a common practice with (often unnecessary) antibiotics prescribed for $44 \%-97 \%$ of hospitalised patients [18][19]. Begum et al. conducted a study in Bangladesh and found that that $38 \%$ of patients received antibiotics (38\%), of which $14 \%$ of prescriptions were irrational [9]. That study highlighted existing malpractice in the use of antibiotics. Baktygul et al. also reported inappropriate use of antibiotics (73.3\%) in a hospital in the Kyrgyzstan Republic, with significantly higher inappropriate antibiotics choice in gynaecology $(\mathrm{OR}=2.70,95 \% \mathrm{Cl}$ : 1.02-7.69) compared with other wards [18]. We aimed to determine if the situation was similar in South Sudan.

Chem et al. evaluated prescribing patterns and predictors of antibiotic prescription in primary healthcare facilities in Cameroon [20]. The overall prescription rate was $36.71 \%$, with a mean of 1.14 antibiotic prescriptions per patient; all prescribed antibiotics were broad-spectrum. That study also showed $98.36 \%$ of medicines were prescribed by generic name and $99.87 \%$ were from the essential drug list. Those authors concluded there was misuse of antibiotics in primary care facilities and recommended prescriptions should only be made by physicians because of their adequate training.

Antibiotics account for a substantial proportion of hospital drug expenditure, and misuse or overuse generates unnecessary costs [15]. Saha et al. [21] noted that the inappropriate prescribing of antibiotics was influenced by knowledge deficits among prescribers, practice environment, practitioner's prior experience, peer pressure, patient pressure, patient expectations, time constraints, diagnostic uncertainty and lack of/ineffective communication between prescribers, pharmacists and patients. Shafiq, et al. [22], found the implementation of treatment guidelines for commonly occurring infections in the tertiary care hospital setting increased the appropriate use of antibiotics. The WHO argued that many prescribers in developing countries have little access to good quality information about diagnosis and drugs. For example, the South Sudan Gold Standards for Treatment (STG) are often unavailable and health workers are often unsupported and unsupervised [10]. These findings confirmed the rationale for this study. 
Healthcare costs for Antibiotic resistant infections are much higher than those for patients with nonresistant infections. Another study noted that common infectious diseases (e.g., tuberculosis [TB], sexually transmitted infections, acute respiratory infections, dysentery, malaria and HIV/AIDS) are becoming increasingly challenging and expensive to treat, with the burden highest in developing countries where resources are limited, and rates of infection are high [23]. In Africa, AMR is an acknowledged problem for HIV and other pathogens that cause malaria, TB, typhoid, cholera, meningitis, gonorrhoea and dysentery [24].

Worldwide, few countries (4.3\%) have national AMR plans, only $14.9 \%$ have national infection prevention control policies, $93.6 \%$ have essential medicines lists and $91.5 \%$ have national medicines policies and treatment guidelines reflecting rational use. However, no countries have national surveillance systems that routinely generate representative, robust data on antimicrobial use and resistance [6]. In response to calls for urgent action, the World Health Assembly) adopted a Global Action Plan on AMR in May 2015. Following this and to meet Africa-specific needs, the Africa CDC established the Anti-Microbial Resistance Surveillance Network (AMRSNET). The AMRSNET goals for the subsequent 5 years were to: improve surveillance of AMR organisms among humans and animals; delay AMR emergence; limit AMR transmission; and mitigate harm among patients infected with AMR organisms.

Ampaire and colleagues reported high levels of AMR to commonly-used antibiotics (ampicillin and cotrimoxazole) in East Africa, with resistance being 50\%-100\% [25]. Among Gram-positive infections, extensive resistance was reported to ampicillin (100\%), gentamicin and ceftriaxone (50\%-100\%) [25]. In South Sudan, no study has been conducted to guide government policy on antibiotics use, and no data are available on antibiotic use. Despite the existence of protocols guiding the use of medicines in the healthcare system, there are reports of patients being prescribed antibiotics and other medicines without microbiological testing. In addition, prescribers do not follow available guidelines or protocols in their day-to-day practice, resulting in incorrect prescribing and use of antibiotics. Therefore, it is necessary to assess the use of antibiotics in South Sudan to provide scientific evidence of the magnitude of this problem. Addressing the problem of AMR is urgent because new antibiotic-resistant mechanisms are emerging that threaten our ability to treat common bacterial diseases and result in prolonged illness, increased treatment costs, disability and death. This study will inform policymakers, health professionals and prescribers regarding implementing strategies to help contain antibiotics resistance in the population.

\section{Problem Statement}

The global pattern of AMR necessitates international guidelines for the use of antibiotics in preventing and treating common infectious diseases. No studies have been conducted in South Sudan to evaluate whether antibiotics are prescribed according to recommended hospital formulas and international guidelines. The paucity of evidence on antibiotic prescribing patterns means there is a knowledge gap regarding the use/misuse of antibiotics in the country. Irunde et al. noted that measuring the level of 
rational antibiotic prescribing helps understand understanding the correct use of antibiotics in a country to prevent development of AMR [14].

\section{Research Questions}

Given the paucity of literature on the use of antibiotics in Africa in general and South Sudan in particular, how can we appropriately plan for antibiotics use and control of AMR in South Sudan? Are antibiotics prescribed according to recommended hospital formulas and standard treatment guidelines in Juba Teaching Hospital (JTH), South Sudan, to reduce the potential for AMR?

\section{Primary Aim}

This study aimed to explore the divergence in the patterns of antibiotics prescription in JTH from established standards, which places the South Sudan population at risk for AMR.

\section{Specific Objectives}

The objectives of this study were:

1. To determine the prevalence of antibiotic use in JTH.

2. To evaluate whether antibiotic treatment in JTH complied with national and international treatment guidelines.

3. To identify the appropriate use of antibiotics in terms of correct choice, dose, duration and route of administration.

\section{Methodology}

This study explored the magnitude of over- and under-prescription of antibiotics at JTH to estimate the risk for AMR. We defined appropriate antibiotic use as use that was consistent with national treatment guidelines. Indications for the prescribed antibiotics were analysed to evaluate compliance with the STG.

\section{Study Design}

This study used a retrospective cross-sectional design. Data were obtained from a review of files for patients admitted to the medical or surgical wards at JTH between January and December 2016. We used non-probability purposive sampling to select files for review.

\section{Study Setting}

This study was conducted at JTH in Juba County, Jubek State (Former Central Equatoria State), which is the capital city of the Republic of South Sudan. JTH is a public tertiary-care institution offering some medical specialities with an official capacity of 260 beds. The 2016 hospital records indicated the hospital had 40,251 admissions in 2016. 


\section{Study Population}

The population for this study included all patients who had been prescribed antibiotics as inpatients irrespective of their condition. Files extracted from the hospital archives included files for patients admitted to JTH medical or surgical wards between January and December 2016. We excluded files with incomplete data.

\section{Sample Size and Description}

The target population was children (aged $0-18$ years) and adults (aged $>18$ years) admitted to JTH medical and surgical wards from January to December 2016. We reviewed files for 316 patients: 113 males and 203 females. The required sample size for our study was estimated with the StatCalc Epi Info ${ }^{\text {TM }}$ calculator as 384 files (95\% confidence level) [26].

\section{Data Collection}

Files were drawn from each ward using probability-proportional to the ward size (Appendix C). The proportion for each month was calculated based on the number of admissions per month divided by the total number of admissions for the year and multiplied by 100 . Each month had a specific sample size determined by multiplying the percentage proportion of that month by the total sample size allocated for the ward. The files required for review were then identified from the ward files using a systematic simple random sampling method determined by the sampling interval. Secondary data were collected from the selected files. Data were collected using a standard data collection form over a 2-month period [18].

\section{Procedure}

Data were collected by a team of data collectors with medical backgrounds who first received two days of training and orientation on how to use the data collection form. The data collected were compiled in a computer database and cleaned by assigning codes. The age, gender, diagnosis, hospital stay, ward, variables for quality assessment and antibiotics prescribed for each patient were coded. Records were reviewed to determine whether the dose, route of administration, duration and correct choice of antibiotics were recorded in the patient's file. Indications for antibiotic use were evaluated by adherence to local guidelines.

\section{Data Analysis and Interpretation}

The criteria developed by Baktygul et al. (Appendix B) were used to assess the appropriateness of antibiotic use in the reviewed files [18]. Prescribed antibiotics were assessed for correctness/incorrectness of choice, duration of therapy, dosage and route of administration. Correctness or appropriateness implied antibiotic choice, duration of therapy, dosage and route of administration were all correct. The data collected for prescribed antibiotics were compared with the STG and standard textbooks to evaluate correct antibiotic use [27][28]. The collected data were then edited, coded, tallied 
and cleared. Double entry and consistent checks were used to minimise potential errors. Descriptive statistics were calculated to meet the study objective.

Data analysis was performed using STATA version 13.0 [29]. Data for categorical variables were coded with numbers. Descriptive statistics (e.g. frequency and percentage) were used to present quantitative data. Results were also presented using tables and figures.

\section{Results}

The primary aim of this study was to estimate the magnitude of antibiotics abuse and misuse in JTH using national treatment guidelines to estimate the risk for AMR. Medical records were reviewed to identify the use of antibiotics during hospital stay for patients hospitalised in 2016. To understand the level of misuse, we examined patients' characteristics, the type of antibiotics used and the clinical conditions for which they were prescribed.

\section{Patients' Demographic and Clinical Characteristics}

About $64 \%$ of participants were female, and over half were aged $\geq 18$ years (Table 1 ). The distribution of patients was non-homogenous in the selected wards, with the medical ward having the most admissions (78.5\%). There were more female than male patients in both wards.

Table 1

Demographic and clinical characteristics of patients at Juba Teaching Hospital (2016)

\begin{tabular}{|lll|}
\hline Variable & Frequency (n) & Percentage (\%) \\
\hline Gender & & \\
Male & 113 & 35.76 \\
\hline Female & 203 & 64.24 \\
\hline Age group, years & \\
\hline $0-5$ & 94 & 29.75 \\
\hline $6-17$ & 25 & 7.91 \\
\hline$\geq 18$ & 197 & 62.34 \\
\hline Ward & & \\
\hline Medical & 248 & 78.48 \\
\hline Surgical & 68 & 21.52 \\
\hline
\end{tabular}

Conditions for which Antibiotics were Prescribed 
Patients were administered antibiotic regimens in both wards for infections in all organ systems. Most patients were diagnosed with infectious and parasitic diseases $(n=75,23.7 \%)$, including diseases of the digestive system $(n=63 ; 19.9 \%)$ and respiratory system $(n=56 ; 17.7 \%)$ (Fig. 1).

\section{Antibiotic Use during the Study Period}

Eight different antibiotic groups and 21 single antibiotics were prescribed in the studied wards (Tables 2 and 3). The majority of prescribed antibiotics (34\%) were from the penicillin group, followed by $23 \%$ from the cephalosporin and $20 \%$ from the metronidazole groups. Only $11 \%$ of prescribed antibiotics were from the aminoglycoside group.

Table 2

Most Commonly Prescribed Antibiotic Groups

\begin{tabular}{|lll|}
\hline Antibiotic $^{\text {a }}$ & Frequency (n) & Percentage (\%) \\
\hline Penicillin & 223 & 34 \\
\hline Cephalosporin & 152 & 23 \\
\hline Metronidazole & 132 & 20 \\
\hline Aminoglycoside & 75 & 11 \\
\hline Quinolone & 38 & 6 \\
\hline Macrolide & 20 & 3 \\
\hline Other & b & 2 \\
\hline Tetracycline & 13 & 1 \\
\hline Total & 7 & 100 \\
\hline a Grouping based on the Anatomical Therapeutic Chemical Classification System. \\
b Other antibiotics included TMP-SMX, chloramphenicol and nitrofurantoin. \\
\hline
\end{tabular}

The frequency of the single antibiotics prescribed is shown in Table 3. In total, there were 660 prescriptions for single antibiotics across the studied wards, with ceftriaxone the most commonly prescribed antibiotic $(21.2 \%)$, followed by metronidazole and amoxicillin ( $20.0 \%$ and $11.5 \%$, respectively). 
Table 3

Prevalence of single antibiotic use at Juba Teaching Hospital (2016)

\begin{tabular}{|lll|}
\hline Antibiotic (single) & Frequency (n) & Percentage (\%) \\
\hline Ceftriaxone & 140 & 21 \\
\hline Metronidazole & 132 & 20 \\
\hline Amoxicillin & 76 & 12 \\
Gentamicin & 74 & 11 \\
Benzylpenicillin & 71 & 11 \\
Others & 167 & 25 \\
\hline Total & 660 & 100 \\
\hline
\end{tabular}

\section{Prevalence of Antibiotic Use by Ward}

Antibiotic use by ward type is shown in Table 4. We found that $75 \%(n=497)$ of antibiotics were prescribed for 248 patients in the medical ward, and 25\% $(n=163)$ of antibiotics were prescribed for 68 patients in the surgical ward. 
Table 4

Prevalence of antibiotic use by ward at Juba Teaching Hospital (2016)

\begin{tabular}{|llll|}
\hline Antibiotic & Medical & Surgical & Total \\
\hline Ceftriaxone & 105 & 35 & 140 \\
\hline Metronidazole & 82 & 50 & 132 \\
\hline Amoxicillin & 53 & 23 & 76 \\
\hline Gentamicin & 70 & 4 & 74 \\
\hline Benzyl penicillin & 70 & 1 & 71 \\
\hline Ampiclox & 21 & 25 & 46 \\
\hline Ciprofloxacin & 32 & 5 & 37 \\
\hline Ampicillin & 9 & 12 & 21 \\
\hline Cefixime & 11 & 0 & 11 \\
\hline Septrin (TMP-SMX) & 10 & 0 & 10 \\
\hline Others & 34 & 8 & 42 \\
\hline Total & 497 & 163 & 660 \\
\hline Cases & 248 & 68 & 316 \\
\hline
\end{tabular}

\section{Number of Antibiotics Prescribed Per Patient during the Admission Period}

During their admission period, $40.2 \%$ of patients received two antibiotics, $30.1 \%$ received one antibiotic and $20.6 \%$ received three antibiotics per prescription (Fig. 2).

Table 5 shows that the average number of antibiotics prescribed per patient encounter was 2.09 (95\% Cl: 1.98-2.19).

Table 5

Average number of antibiotic prescriptions per patient at Juba

Teaching Hospital (2016)

\begin{tabular}{|llll|}
\hline Variable & Mean & Std. error & 95\% Confidence interval \\
\hline Antibiotic & 2.094937 & 0.0533348 & $1.989999-2.199874$ \\
\hline Medical & 2.012097 & 0.0600601 & $1.893927-2.130266$ \\
\hline Surgical & 2.397059 & 0.1091505 & $2.182303-2.611815$ \\
\hline
\end{tabular}

Quality Assessment of Antibiotic Therapy 
Choice of Drug

We assessed the correct and incorrect choice of antibiotics prescribed for each patient. A correct choice of antibiotics for therapy was observed in $29.43 \%(n=93)$ of the reviewed files; however, incorrect use was noted in $70.57 \%(n=223)$ of the files.

Dose Documentation

A correct dose of the prescribed antibiotics was documented in 281 (88.9\%) files, and incorrect doses were noted for $35(11.1 \%)$ patients.

\section{Dosage Form}

The most commonly used administration method was injection solution (45.2\%), followed by tablet (25.2\%) and capsule (17.3\%) (Table 6).

Table 6

Modes of Antibiotic Delivery

\begin{tabular}{|lll|}
\hline Dosage form & Number $(\mathbf{n})$ & Percentage (\%) \\
\hline Syrup/suspension & 62 & 12.00 \\
\hline Capsule & 89 & 17.30 \\
\hline Tablet & 130 & 25.20 \\
\hline Injection solution & 233 & 45.20 \\
\hline Other & 2 & 0.40 \\
\hline Total & 516 & 100.00 \\
\hline
\end{tabular}

Duration of Therapy

As shown in Table 7, 74.1\% of patients received antibiotic therapy during an admission period of $0-7$ days, $21.5 \%$ of patients were treated in an $8-14$-day admission period and $4.4 \%$ of patients received antibiotic treatment in an admission period $\geq 15$ days. 
Table 7

Duration of therapy for patients admitted to Juba

Teaching Hospital (2016)

\begin{tabular}{|lll|}
\hline Admission period & Number $(\mathrm{n})$ & Percentage (\%) \\
\hline $0-7$ days & 234 & 74.10 \\
$8-14$ days & 68 & 21.50 \\
$\geq 15$ days & 14 & 4.40 \\
\hline Total & 316 & 100.00 \\
\hline
\end{tabular}

Route of Administration

Review of the single antibiotics used showed the majority of antibiotics were given intravenously (51.5\%), followed by oral (47.6\%) and intramuscular (0.66\%) administration (Fig. 3). In addition, parenteral antibiotics were used more often than oral antibiotics.

\section{Rationality of Antibiotic Therapy at JTH}

\section{Appropriateness of Antibiotic Prescription}

Antibiotic therapy was correctly used (appropriate) in $21.2 \%(n=67)$ of the prescriptions, and incorrect (inappropriate) in $78.8 \%(n=249)$. Figure 4 provides a graphical summary of Baktygul et al.'s criteria for antibiotic use [18].

Antibiotics prescribed by diagnosis were evaluated for compliance with local treatment guidelines. Only $29.11 \%(n=92)$ of prescriptions complied with treatment according to these guidelines, and $70.89 \%(n=$ 224) did not comply (Fig. 5).

\section{Discussion}

The primary aim of this study was to explore the prevalence of antibiotic misuse in South Sudan, which exposes the population to risk for AMR. The results indicated that there was over-prescription of antibiotics in South Sudan. However, it remains unclear if this translates to overuse or underuse given the socio-economic conditions. There are also government regulatory issues related to availability of overthe-counter antibiotics in the study setting, and it remains unclear whether healthcare personnel were aware of AMR.

Few studies focused on AMR have been conducted in Africa, and none in the South Sudan setting. This study evaluated how antibiotics were used to fill this knowledge gap and elucidate how this prescribing practice/pattern contributed to the development of AMR. In the African region, understanding of issues related to AMR and its magnitude is hampered by the fact that surveillance of drug resistance is limited to a few countries, resulting in incomplete and inadequate data on the true extent of the problem [30]. 
Despite considerable improvement in the availability and control of antibiotics in hospitals, rational antibiotic use remains a worldwide concern [31]. If this issue is not addressed, the future may resemble the days before the development of the 'miracle' drugs, as described by [32]. That is, an era where people die of common infections (such as during surgery, chemotherapy, organ transplantation and care for premature infants) and medical intervention becomes impossible.

This study demonstrated high antibiotics use at JTH, with antibiotics commonly being used inappropriately (78.8\%). Non-adherence to local guidelines was particularly high (70.9\%). The proportion of antibiotics prescribed at JTH was 100\%, with an average of 2.1 (95\% Cl: 1.98-2.19) antibiotics per patient prescription. This study also found that infectious and parasitic diseases were the most prevalent conditions for which antibiotics were prescribed, and there was a high percentage of intravenous antibiotics used (51.5\%). Our finding that the prescribed antibiotics were inappropriate in $78.8 \%$ of cases was consistent with studies from Indonesia (78.5\%), Ethiopia (80.6\%) and the Kyrgyz Republic (73.3\%). The literature suggests that $44-97 \%$ of all antibiotic prescriptions in hospitals globally are inappropriate [19], especially in developing countries [18]. When comparing the level of inappropriate antibiotic prescription between developed and developing countries (e.g. Switzerland vs. Ethiopia), it becomes evident that developing countries are far behind in terms of implementing strategies to curb antibiotic use and resistance. A possible explanation for differences between developed and developing countries is a lack of local antibiotic prescribing policies and strategies to control the misuse/overuse of antibiotics in developing countries. It has been suggested that implementing treatment guidelines for the most commonly occurring infections in hospitals may increase appropriate use of antibiotics.

In this study, the most commonly used antibiotic group was penicillin (33.8\%). This was consistent with a study conducted in Swiss hospitals by [15], where the penicillin group were the most commonly prescribed (33\% of cases). However, this differed from studies from Ethiopia [33] East Africa [34] and Egypt [35] in which cephalosporins were the most used antibiotics (32.7\%, 34\% and $28.7 \%$ respectively). Penicillin and cephalosporin are the two most commonly prescribed antibiotics groups because of their broad spectrum of activity.

The most frequently used single antibiotic in JTH was ceftriaxone (21.2\%). This result was comparable with studies by Mbam et al. [36] and Abrha et al. [33] in Cameroon (30\%) and Ethiopia (28.7\%), respectively. However, different antibiotics were commonly used in Jordan [37] and Turkey [38], with imipenem/cilastatin (41.25\%) and amoxicillin (29.9\%) being the most used antibiotics, respectively. Findings from previous studies indicated that prescribers tended to prescribe higher antibiotics, particularly third-generation cephalosporin, disregarding adherence to national treatment guidelines. Evidence suggests that treatment with second- or third-line drugs may be less effective, more toxic and more expensive [19].

We found that a number of inpatient records documented three $(20.6 \%)$ or four (8.54\%) antibiotics prescribed in a single encounter, with an average of 2.1 (95\% Cl: 1.98-2.19) antibiotics per prescription. This finding was comparable with studies from East Africa [34] and Ghana [39], where two antibiotics 
were prescribed per patient encounter on average. However, it was inconsistent with studies conducted in the Kingdom of Saudi Arabia [40] and Cameroon [20] where a single antibiotic was recorded per prescription. The difference between those studies and this study may reflect a need for more time to diagnose the patient and select the appropriate treatment.

Doctors may overprescribe antibiotics because of lack of knowledge about rational use or because they want to prevent potential infections. However, it is advisable to keep the number of antibiotics per encounter as low as possible to minimise the risk for drug interaction and development of bacterial resistance. The 2.1 antibiotics per encounter found in this study indicated there is a need for improvement with regard to polypharmacy, especially given the WHO recommended average of 1.6-1.8 antibiotics per encounter.

The most common clinical indications for antibiotic use in this study were infectious and parasitic diseases ( $n=75,23.7 \%)$. However, these findings were inconsistent with studies from Egypt [35] and Jordan [37], where the most prevalent conditions included respiratory tract infections (39.2\%) and urinary tract infections (53.75\%). A study from India revealed that $69.4 \%$ of patients received antibiotics for acute respiratory infection and diarrhoea of viral origin [9]. This suggested that the burden of infectious and parasitic diseases in South Sudan contributed to the overuse of antibiotics. The increased burden of infections in developing countries may therefore explain the misuse of antibiotics in healthcare settings associated with AMR.

In this study, the majority of antibiotics were given intravenously $(51.5 \%)$ followed by oral $(47.6 \%)$ and intramuscular $(0.66 \%)$ administration, with overall injectable antibiotics constituting $45.2 \%$. Intravenous administration, including injectable antibiotics, exceeded the optimal level recommended by the WHO of $\geq 10 \%$ [14]. In a similar study conducted in Jordan, $47.5 \%$ of patients were prescribed intravenous antibiotics [37]. However, the proportion in our study was lower compared with a similar study in Pakistan, where injections accounted for 75\% [41]. However, the presence of paediatric patients in our study population might have contributed to the high use of intravenous antibiotics. It has been reported that intravenous administration is associated with risks for transmission of blood-borne infections including hepatitis B/C and HIV/AIDS [14]. Moreover, shifting the route of administration from an intravenous to an oral route saves costs, shortens the length of hospital stay and decreases adverse reactions from intravenous use [33].

In this study, $74.1 \%$ of patients received antibiotic therapy during a $0-7$-day admission period, whereas $21.5 \%$ of patients received antibiotic treatment over $8-14$ days and $4.4 \%$ of patients received treatment for a period exceeding 2 weeks. Evidence shows that the cost of healthcare for patients with resistant infections is high because of a longer duration of illness, additional tests and use of more expensive drugs [10].

\section{Limitations of this Study}


Our findings showed that the irrational use of antibiotics and non-adherence to national treatment guidelines were high at JTH. However, our findings might not reflect the actual picture of antibiotics prescribing in South Sudan because we only included files for patients admitted to two JTH wards, which is a tertiary level hospital in the capital city. The other studies discussed above might not have been conducted in tertiary level settings. In addition, the sample in this study might not be representative of the whole South Sudan population. Furthermore, despite receiving training, the data collectors involved in this study might not have been sufficiently knowledgeable.

Challenges were encountered during the assessment process. First, the level of antibiotic use could not be quantified because of lack of medication charts, and a lack of local data on antibiotic use and resistance. Second, prescribers had poor handwriting making it difficult for data collectors to obtain the correct information. Third, the quality of organisation and record-keeping in the study area was poor. Fourth, it was not possible to investigate relationships between the adequacy/inadequacy of treatments and clinical outcomes. Finally, Baktygul et al. method was used to evaluate the quality of antibiotics use [18]. Although this method is a standard method of evaluating prescribing antibiotics used in several studies, it may have limitations depending on the location and medical facilities.

\section{Conclusion And Recommendations}

This study explored antibiotic prescribing practices in a hospital in South Sudan. Antibiotic use in JTH is seriously inappropriate $(78.8 \%)$, with prescribing patterns failing to adhere to the national guidelines (70.9\%). The proportion of antibiotics prescribed is $100 \%$, with an average of 2.1 antibiotics per patient prescription. The rate of intravenous antibiotics use (51.5\%) observed in the study area is high. This study also found that most antibiotics are prescribed for infectious and parasitic diseases.

Given shortcomings in the development of new generations of antibiotics, appropriate use of antibiotics is critical to ensure the availability of effective treatment for bacterial infections in hospitals. It is envisaged that our findings may reflect the real picture of antibiotic use in JTH and contribute to knowledge in this area. Furthermore, this study is expected to fill a gap in terms of the lack of current data on antibiotic prescribing patterns and resistance in South Sudan.

Based on the findings of this study, the following recommendations have been suggested as possible ways to address the problem of AMR arising from drug misuse or overuse.

- The South Sudan Ministry of Health should adopt national surveillance systems that routinely generate representative, robust data on antibiotic use and resistance to monitor antibiotic dispensing.

- Health professionals should adopt international standard and locally appropriate guidelines for antibiotic use in hospitals and primary healthcare centres.

- Healthcare institutions should introduce programmes to improve rational antibiotic use, initiate education campaigns, regulate drug auditing practices, restrict dispensing techniques and implement 
controls.

- Further research is needed to examine the associations between antibiotics and their appropriate use.

\section{Declarations}

\section{Ethics approval and consent to participate}

This manuscript was approved by the Ministry of Health Ethics Review Board and given approval number $\mathrm{MOH} / \mathrm{ERB} 51 / 2018$. The study hospital was formally contacted and consent sought for their participation. The invitation letter included the detailed study protocol and a statement outlining the study purpose, potential benefits and an estimated time for completion.

\section{Consent for publication}

All authors consented to the publication of the study

\section{Availability of data and materials}

The datasets generated and/or analysed during the present study (which include individual treatment files) are not publicly available because of ethical restrictions but may be available from the corresponding author on reasonable request.

\section{Competing interests}

The authors declare that they have no competing interests.

\section{Funding}

None to declare.

\section{Authors' contributions}

MEO and DKD led the conception and design of the study, reviewed the files, generated data for analysis and interpretation, and wrote the manuscript. JPM and AMA read the draft, reviewed the findings and worked on the manuscript. All authors critically analysed the paper for important intellectual content. All authors read and approved the final manuscript.

\section{References}

1. Interagency Coordination Group (IACG) on Antimicrobial Resistance. (2019, Apr.). No Time to Wait: Securing the Future from Drug-Resistant Infections. Report to the Secretary General of the United Nations. https://www.who.int/antimicrobial-resistance/interagencycoordinationgroup/IACG_final_report_EN.pdf?ua=1. 
2. O'neill JTackling. Drug-Resistant Infections Globally: Final Report and Recommendations, May 2016. Available online at: http://amrreview.org/sites/default/files/160525_Final\%20paper_with\%20cover.pdf). [Accessed on 10 December 2018].

3. Gould IM, Bal AM. New antibiotic agents in the pipeline and how they can help overcome microbial resistance. Virulence. 2013;4(2):185-91. [PMC free article] [PubMed] [Google Scholar].

4. Bartlett JG, Gilbert DN, Spellberg B. Seven ways to preserve the miracle of antibiotics. Clin Infect Dis. 2013;56(10):1445-50. [PubMed] [Google Scholar.

5. Founou, et al. Antibiotic Resistance in the Food Chain: A Developing Country-Perspective. Front Microbiol. 2016;7:1881. doi:10.3389/fmicb.2016.01881.

6. Essack, et al. Antimicrobial resistance in the WHO African region: current status and roadmap for action. J Pub Health. 2016;39:8-13.

7. Asante KP, et al. Knowledge of antibiotic resistance and antibiotic prescription practices among prescribers in the Brong Ahafo Region of Ghana. BMC Health Services Research. 2017;17:422.

8. World Health Organization (WHO). The rational use of drugs: Report of the Conference of Experts Nairobi, 25-29 November 1985, Geneva 1987.

9. Begum T, et al. An audit of rational use of antibiotics in a tertiary hospital of Bangladesh. Delta Med Col J Jul. 2014;2(2):64-7.

10. WHO (World Health Organisation). Essential Drugs Monitor, World Health Organization $\mathrm{CH}-1211$ Geneva 27. Switzerland Double Issue - No. 2000;28:\& 29.

11. Zaman SB, et al., A Review on Antibiotic Resistance: Alarm Bells are Ringing. Cureus 9(6): e1403. DOI 10.7759/cureus.1403.

12. Centers for Disease Control and Prevention (CDC). Office of Infectious Disease, Antibiotic resistance threats in the United States, 2013. Available online at http://www.cdc.gov/drugresistance/ threatreport-2013 [Accessed on 19 September 2019].

13. Tadesse BT, et al. Antimicrobial resistance in Africa: a systematic review. BMC Infect Dis. 2017;17:616.

14. Irunde $\mathrm{H}$, et al. Assessment of rational medicines prescribing in health-care facilities in four regions of Tanzania. J Pharm Prac Community Med. 2017;3(4):225-31.

15. Bugnon-reber AV, et al. Antibiotic misuse in medium-sized Swiss hospitals. Swiss Med Wkly. 2004;134:481-5. Available online at www.smw.ch [Accessed on 3 April 2019].

16. Ashraf S, Cook P. Antibiotic misuse in hospital, outpatient, and long-term care settings. N C Med J. 2016;77(5):346-9.

17. Siddiqi S, et al. Prescription practices of public and private health care providers in Attock district of Pakistan. Int J Health Plann Mgmt. 2002;17:23-40.

18. Baktygul $\mathrm{K}$, et al. An assessment of antibiotics prescribed at the secondary health-care level in the Kyrgyz Republic. Nagoya J Med Sci. 2011;73:157-68. 
19. Abdullah R. Antibiotic Abuse in Developing Countries. Pharmaceut Reg Affairs 1:e106. doi:10.4172/2167-7689.1000e106.

20. Chem ED, et al., 2018. Prescribing patterns and associated factors of antibiotic prescription in primary health care facilities of Kumbo East and Kumbo West Health Districts, North West Cameroon. PLoS ONE 13(3): e0193353. Available online at https://doi.org/10.1371/journal. pone.0193353 [Accessed on 18 July 2019].

21. Saha SK, et al. Improving antibiotic prescribing by general practitioners: a protocol for a systematic review of interventions involving pharmacists. BMJ Open. 2018;8:e020583.

22. Shafiq N, et al. Antibiotic stewardship in a tertiary care hospital of a developing country: establishment of a system and its application in a unit-GASP Initiative. Infection. 2016;44:651-9. https://doi.org/10.1007/s15010-016-0913-z.

23. MSH. 2012. Strengthening Pharmaceutical Systems. How to investigate antimicrobial use in hospitals: Selected indicators. Management Sciences for Health, Arlington, VA.

24. CDC (Centres for Disease Control) Africa., 2019. Framework for antimicrobial resistance, 2018-2023 AU. Available online at www. Africa_CDC_AMR_Framework_Eng.pdf [Accessed 12 July 2019].

25. Ampaire L, et al. A review of antimicrobial resistance in East Africa. Afr J Lab Med. 2016;5(1), a432. Available online at http://dx.doi.org/10.4102/ajlm.v5i1.432. [Accessed on 04 August 2019].

26. Epi CDC, Info ${ }^{\text {TM}}$. Division of Health Informatics \& Surveillance (DHIS), Center for Surveillance, Epidemiology \& Laboratory Services (CSELS), https://www.cdc.gov/epiinfo/userguide/statcalc/statcalcandopenepi.html (2019).

27. WHO, South Sudan revises its. 2007 Essential Medicines List to improve standards and access to Essential Medicines within the health care system, https://www.afro.who.int/news/south-sudanrevises-its-2007-essential-medicines-list-improve-standards-and-access-essential (2018).

28. $\mathrm{MOH}$ (Ministry of Health). Government of Southern Sudan. Prevention and Treatment Guidelines for Primary Health Care Centres and Hospitals. http://www.southsudanmedicaljournal.com/assets/files/misc/SS_Treatment_Guidelines07.pdf. (2006).

29. STATA (Version 13). StataCorp LP. 2021, https://stata-13.software.informer.com/ (2021).

30. Ndihokubwayo JB, et al. Antimicrobial resistance in the African region: Issues, challenges and actions proposed. Key Determinants for Health in the African Region. African Health Monitor 2013;16.

31. Agalu A, et al. Antibiotic prescribing pattern in a referral hospital in Ethiopia, Glob. J. Pharm. Pharmacol. 2013.

32. Golker, et al. Antibiotic resistance crisis. J Infect Dev Ctries. 2014;8(2):129-36.

33. Abrha $S$, et al. Antibiotic utilization and their cost in Ayder Referral Hospital, Mekelle, Ethiopia. USA: Global Journals Inc. Volume 15 Issue 1, 2015. 
34. Palikhe N. Prescribing Pattern of Antibiotics in Paediatric Hospital of Kathmandu Valley. Kathmandu University Medical Journal. 2004;2(1):6-1.

35. Talaat $\mathrm{M}$, et al. A point prevalence survey of antibiotic use in 18 hospitals in Egypt. Antibiotics. 2014;3:450-60. . Available online at. [Accessed on 07 August 2019].

36. Mbam LA, et al., Indications and patterns of antibiotic prescription in the Buea Regional Hospital of Cameroon. Health Sci. Dis: Vol 16 (1) Jan-Feb-Mar 2015. Available at. [Accessed on 5 September 2019].

37. Jarab AS, et al. Patterns of antibiotic use and administration in hospitalized patients. in Jordan Saudi Pharmaceutical Journal. 2018;26:764-70. Available online at www.sciencedirect.com [Accessed on 10 August 2019].

38. Acimis NM, et al. The prevalence of prescribing antibiotics by primary health care physicians in Turkey: A multi-centered survey. Pak J Med Sci. 2009;25(5):706-11.

39. Labi AK, et al. Antibiotic use in a tertiary healthcare facility in Ghana: a point prevalence survey. Antimicrobial Resistance Infection Control. 2018;7:15.

40. Alharafsheh A, et al. A retrospective cross-sectional study of antibiotics prescribing patterns in admitted patients at a tertiary care setting in the KSA. International Journal of Health Sciences Vol. 12, Issue 4 (July - August 2018). Available online at https://ijhs.org.sa/index.php/journal/article/view/2629/864. [Accessed on 12 June 2019].

41. Qasim S, Muqeet W. Evaluation of antibiotics use based on various parameters used for selection of antibiotics in Nishter Hospital Multan, Pakistan. J App Pharm Vol. April 2014;6(2):171-83.

\section{Figures}

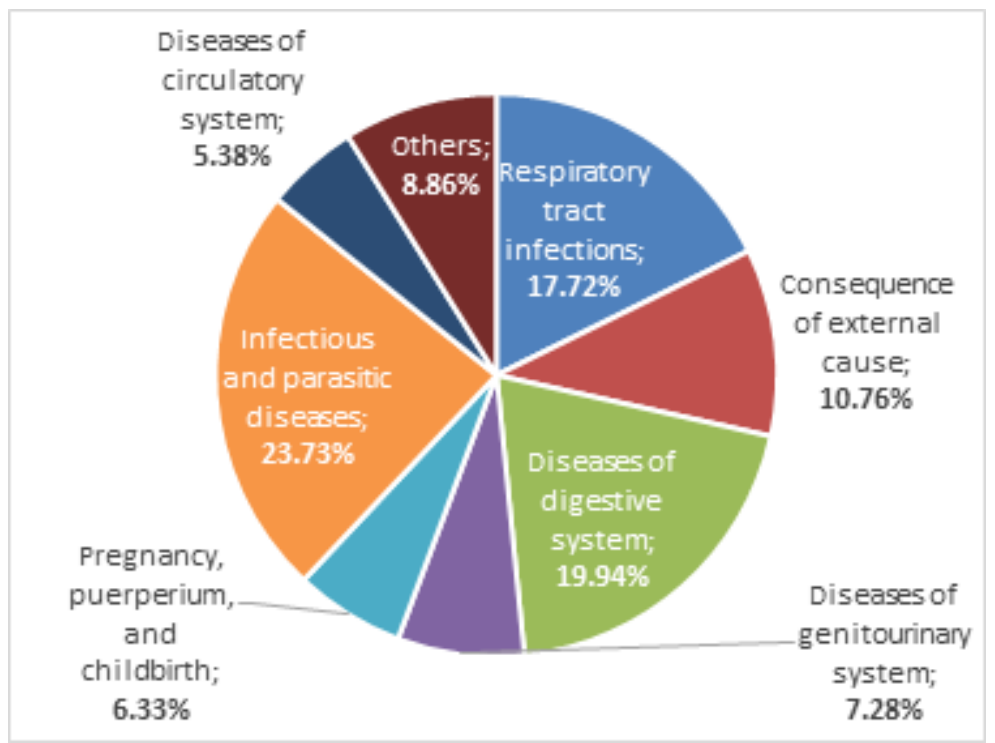

\section{Figure 1}

Diagnoses of patients who were prescribed antibiotics at Juba Teaching Hospital (2016) 


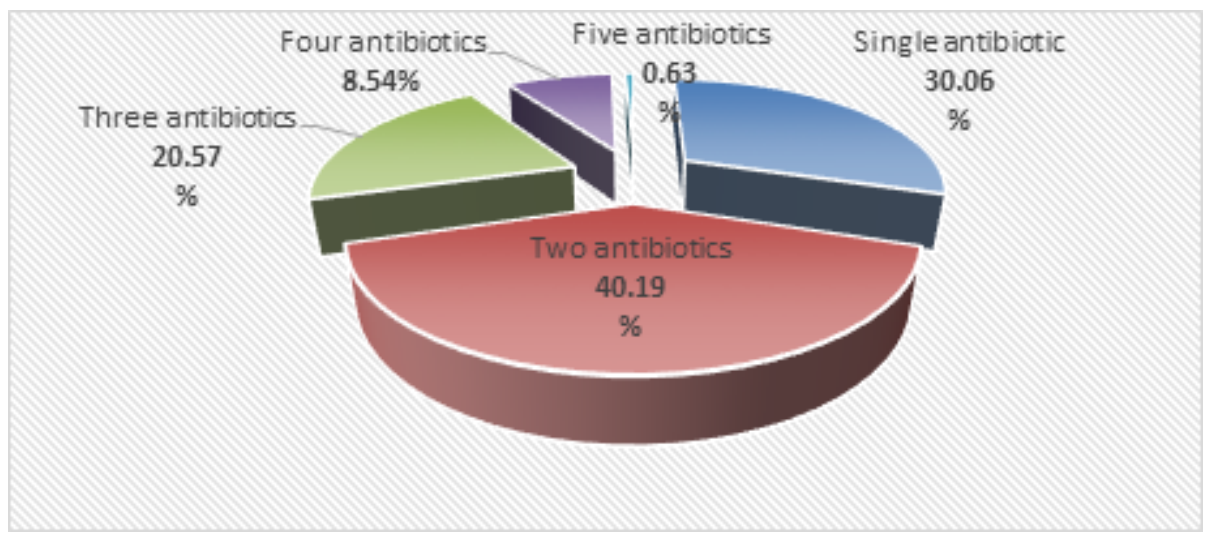

\section{Figure 2}

Number of antibiotics per prescription at Juba Teaching Hospital (2016) $(n=316)$

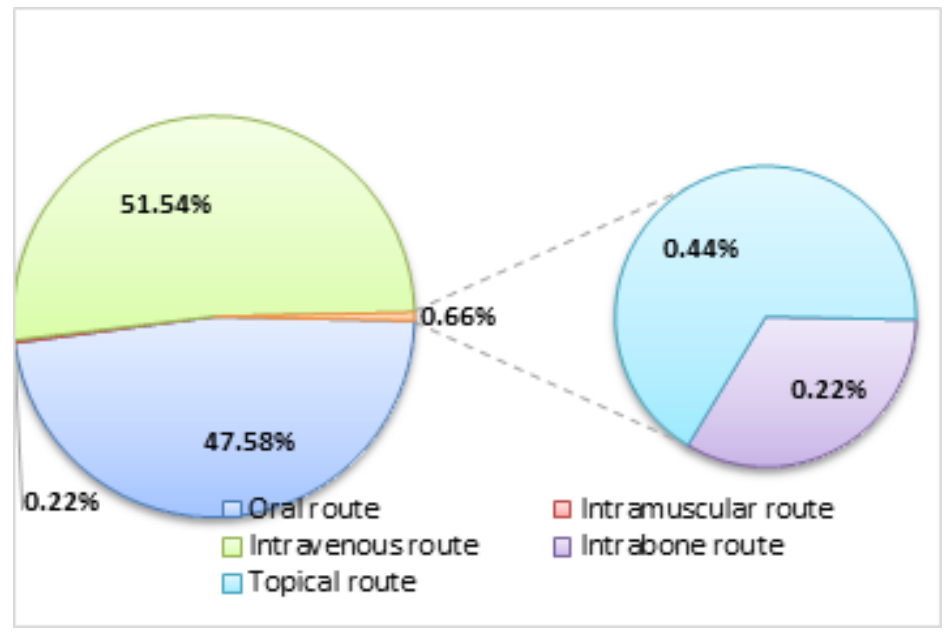

\section{Figure 3}

Route of antibiotics administration $(n=454)$

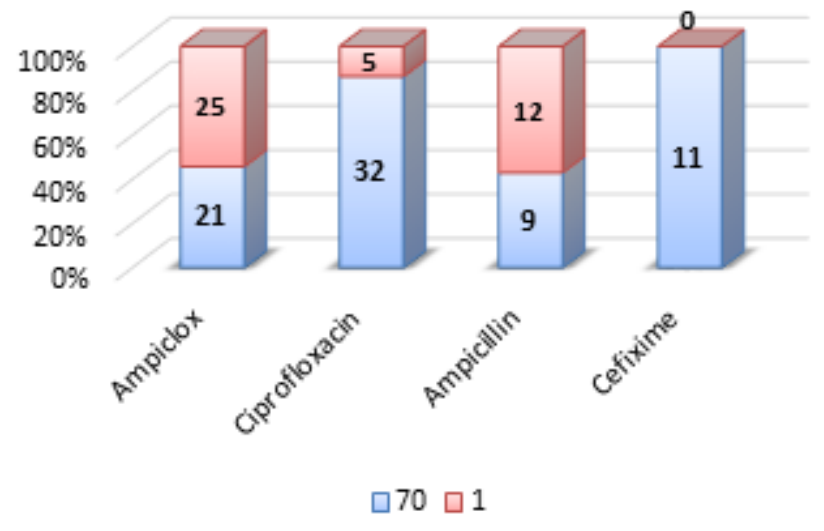

\section{Figure 4}

Antibiotic use based on criteria proposed by Baktygul et al (2011). [18] 


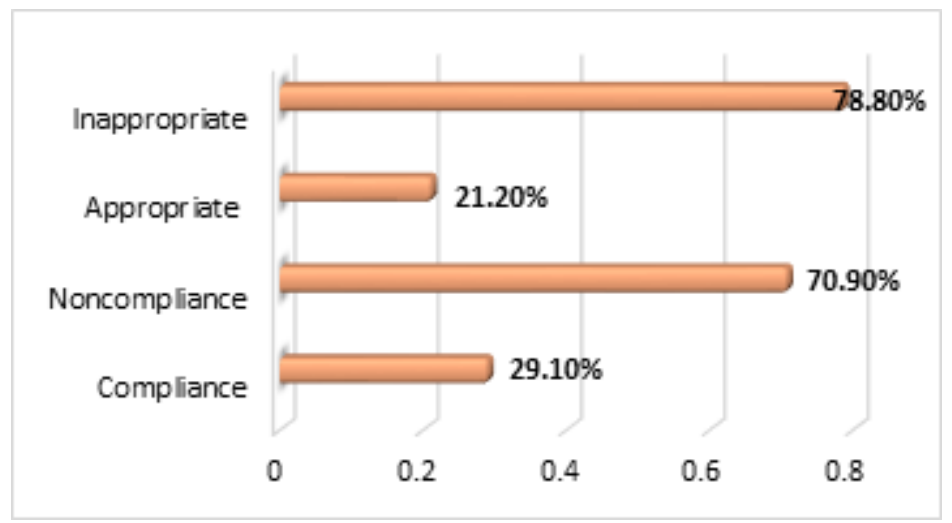

\section{Figure 5}

Appropriateness and compliance of antibiotic use at JTH (2016)

\section{Supplementary Files}

This is a list of supplementary files associated with this preprint. Click to download.

- Appendix.docx 